\title{
Nearly unbiased estimator of contemporary effective mother size using within-cohort maternal sibling pairs incorporating parental and nonparental reproductive variations
}

\author{
Tetsuya Akita $\mathbb{D}^{1}$
}

Received: 7 May 2019 / Revised: 30 August 2019 / Accepted: 5 September 2019 / Published online: 2 October 2019

(c) The Author(s) 2019. This article is published with open access

\begin{abstract}
In this study, we developed a nearly unbiased estimator of contemporary effective mother size in a population, which is based on a known maternal half-sibling relationship found within the same cohort. Our method allows for variance of the average number of offspring per mother (i.e., parental variation, such as age-specific fecundity) and variance of the number of offspring among mothers with identical reproductive potential (i.e., nonparental variation, such as family-correlated survivorship). We also developed estimators of the variance and coefficient of variation of contemporary effective mother size and qualitatively evaluated the performance of the estimators by running an individual-based model. Our results provide guidance for (i) a sample size to ensure the required accuracy and precision when the order of effective mother size is available and (ii) a degree of uncertainty regarding the estimated effective mother size when information about the size is unavailable. To the best of our knowledge, this is the first report to demonstrate the derivation of a nearly unbiased estimator of effective population size; however, its current application is limited to effective mother size and situations, in which the sample size is not particularly small and maternal half-sibling relationships can be detected without error. The results of this study demonstrate the usefulness of a sibship assignment method for estimating effective population size; in addition, they have the potential to greatly widen the scope of genetic monitoring, especially in the situation of small sample size.
\end{abstract}

\section{Introduction}

Contemporary effective population size, which is sensitive to ecological time-scale events, has become recognized as an informative parameter in a focus population, especially in the context of conservation biology and wildlife management (Luikart et al. 2010). There are several methods for estimating contemporary effective population size from genetic markers, such as the temporal method (Nei and Tajima 1981), heterozygote excess method (Pudovkin et al. 1996), molecular coancestry

Supplementary information The online version of this article (https:// doi.org/10.1038/s41437-019-0271-6) contains supplementary material, which is available to authorized users.

Tetsuya Akita

aktiatetsuya1981@affrc.go.jp

1 National Research Institute of Fisheries Science, Japan Fisheries Research and Education Agency, 2-12-4 Fukuura, Kanazawa, Yokohama 236-8648 Kanagawa, Japan method (Nomura 2008), linkage-disequilibrium method (Waples 2006), and kinship assignment method (Wang 2009). At present, it is known that values estimated by these methods display large uncertainties and/or biases under conditions, such as small sample size, small marker numbers, and large effective population size; thus, a widely applicable method is required (Wang et al. 2016; Marandel et al. 2018).

Owing to rapid developments in genotyping technology, a large number of genetic markers, including thousands of genome-wide single nucleotide polymorphisms, have become available for analyzing population structure and demography. As a result, a more accurate estimation of contemporary effective population size can be obtained by, for example, more accurately assigned kinships (Wang et al. 2016). In addition, the recently developed theory of estimation of absolute adult number, which is based on sampled kinship pairs and known as the closekin mark-recapture (CKMR) method (Bravington et al. 2016a, b; Skaug 2017; Hillary et al. 2018), makes it possible to use a full-sibling (FS) or half-sibling (HS) pair; this involves many more DNA markers for detection 
than a parent-offspring pair. It should be noted that the CKMR method is designed to minimize the effect of reproductive variance originating from unmodeled covariates, such as avoiding the use of sibling pairs sampled from the same cohorts; meanwhile, reproductive variance strongly affects the estimation of contemporary effective population size.

Reproductive variance has two components. The first component is variation in age, size, and other factors, which affects average fecundity and originates from differences in life-history parameters (Felsenstein 1971). For example, in the case of teleost species that have a long life span, the number of eggs produced by a mother (i.e., annual fecundity) is determined by her body size; thus, there is considerable variation in reproduction among mothers. The second component is variation in reproduction among parents of the same age or size. An extreme case reflecting this variation is referred to as the "Sweepstakes Reproductive Success (Hedgecock and Pudovkin 2011)," in which only several families reproduce successfully. This phenomenon has received much attention not only for elucidating the ecology of species that display highly variable early life mortality (i.e., type-III life history) but also for providing an opportunity to test the applicability of the multiple-merger coalescent model, a recently developed theory in population genetics (Tellier and Lemaire 2014; Eldon et al. 2016). Addressing the two aforementioned types of variance together can provide insights for interpreting estimated values of effective population size.

In this paper, we propose a new method for estimating the contemporary effective mother size in a population. This approach is based on the number of maternal HS (MHS) pairs found within the same cohort and on modeling that explicitly incorporates overdispersed reproduction, assuming that kinships are genetically detected without any error. Our model partitions reproductive variance into two types of variations: (i) age- or size-specific differences in mean fecundity (referred to as "parental variation") and (ii) unequal contributions by mothers of the same age or size to the number of offspring at sampling (referred to as "nonparental variation") First, we formulate the distribution of offspring number under the two types of variations. Second, we analytically derive the probability that two randomly selected individuals found in the same cohort share an MHS relationship. Third, we determine a nearly unbiased estimator of contemporary effective mother size and its relative estimators. Finally, we investigate the performance of the estimators by running an individual-based model. Our modeling framework may be applied to diverse animal species; however, the description of the model focuses on fish species, which are currently the best candidate target of our proposed method.
Table 1 List of mathematical symbols in main text

\begin{tabular}{|c|c|}
\hline$n$ & Sample number of offspring \\
\hline$n_{\text {pair }}$ & Number of pairs in a sample $\left(={ }_{n} \mathrm{C}_{2}\right)$ \\
\hline$N$ & $\begin{array}{l}\text { Number of mothers in the population when sampled } \\
\text { offspring are born }\end{array}$ \\
\hline$N_{\mathrm{e}}$ & Effective number of mothers in the population \\
\hline$\phi$ & $\begin{array}{l}\text { Overdispersion parameter under negative binomial } \\
\text { reproduction }\end{array}$ \\
\hline$\lambda_{i}$ & $\begin{array}{l}\text { Expected number of surviving offspring of mother } i \text { at } \\
\text { sampling }\end{array}$ \\
\hline$f(\lambda)$ & Frequency of $\lambda$ for all mothers \\
\hline$k_{i}$ & Number of surviving offspring born to mother $i$ \\
\hline$H$ & Number of maternal half-sibling pairs found in samples \\
\hline$\pi$ & $\begin{array}{l}\text { Probability that a randomly selected pair (two offspring) } \\
\text { share a maternal half-sibling relationship }\end{array}$ \\
\hline$c$ & $\begin{array}{l}\text { Combined effect of deviation from the Poisson }\left(=\left(1+\phi^{-1}\right)\right. \\
\left.\mathbb{E}\left[\lambda^{2}\right] / \mathbb{E}[\lambda]^{2}\right)\end{array}$ \\
\hline$\hat{N}_{\mathrm{e}, 0}$ & Moment estimator of $N_{\mathrm{e}}$ \\
\hline$\hat{N}_{\mathrm{e}, 1}$ & Nearly unbiased estimator of $N_{\mathrm{e}}$ \\
\hline$\hat{N}_{\mathrm{e}, \mathrm{TM}}$ & Moment estimator of $N_{\mathrm{e}}$ by the temporal method \\
\hline$\hat{v}$ & Estimator of $\mathbb{V}\left[\hat{N}_{\mathrm{e}, 1}\right]$ \\
\hline$\widehat{c v}$ & Estimator of $\mathbb{C V}\left[\hat{N}_{\mathrm{e}, 1}\right]$ \\
\hline$b_{\text {mean }}$ & Bias of $\hat{N}_{\mathrm{e}, 1}$ \\
\hline$b_{\mathrm{var}}$ & Bias of $\hat{v}$ \\
\hline
\end{tabular}

\section{Theory}

Main symbols used in this paper are summarized in Table 1.

\section{Hypothetical population and sampling scheme}

Here, we suppose that there is a hypothetical population consisting of $N$ mothers and that there is no population subdivision or spatial structure. In this paper, a mature female is referred to as a mother even if she does not produce offspring. For the detection of MHS pairs, $n$ offspring within the same cohort are simultaneously and randomly sampled in the population. For mathematical tractability, we assume that there is only one spawning ground in which the mothers remain for the entire spawning season.

In our modeling framework, if an MHS pair also shares a paternal HS (PHS) relationship, the pair is considered to be an MHS pair (i.e., the FS relationship is assigned as MHS relationship). The technical difficulties of distinguishing an MHS pair from a PHS pair are addressed in the "Discussion" section.

\section{Reproductive potential and its variation (parental variation)}

Here, we introduce the concept of the reproductive potential of mother $i(i=1,2, \ldots, N)$, which is defined as the 


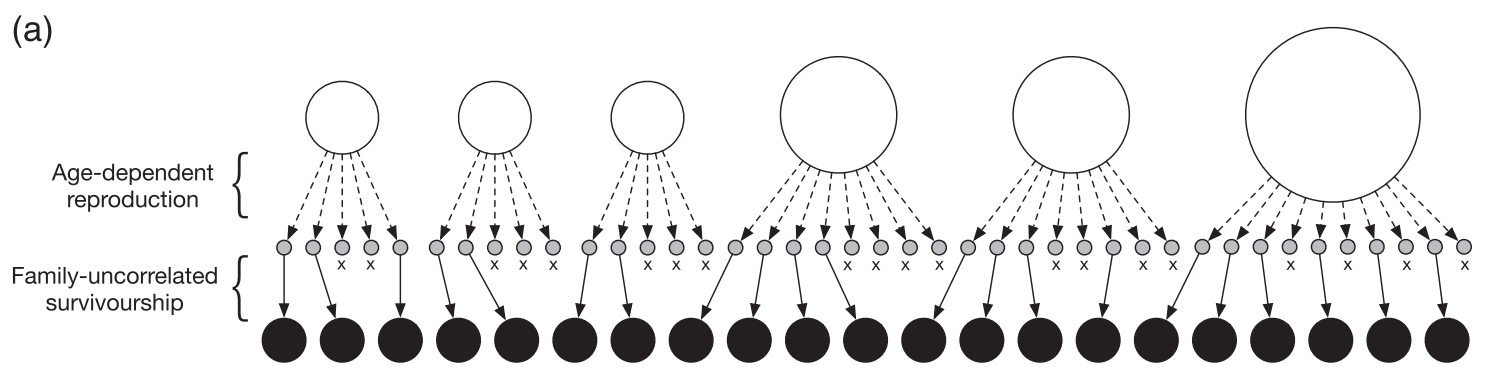

(b)

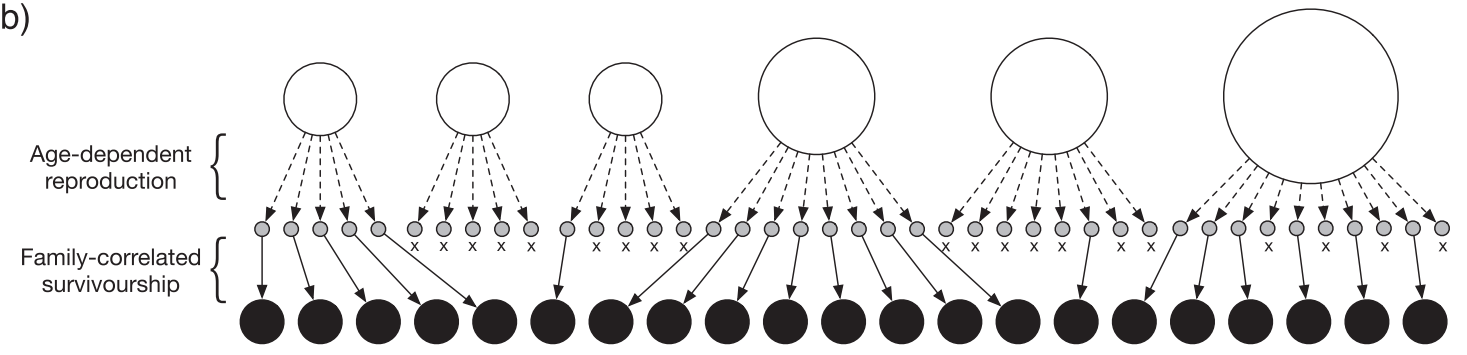

Fig. 1 Example of relationships between mothers and their offspring number for only parental variation (a) and both parental- and nonparental variation (b). $N=6$ and $\sum_{i}^{N} k_{i}=21$. Open, gray, and black circles represent mothers, their eggs, and their offspring, respectively.
The area of an open circle indicates the degree of reproductive potential of each mother (i.e., $\lambda_{i}$ ). Dotted and thin arrows show mother-egg and egg-offspring relationships, respectively. The $\mathrm{x}$ symbol indicates a failure to survive at sampling expected number of surviving offspring at sampling time, denoted by $\lambda_{i}$. The reproductive potential is determined by several factors, including the mother's age, weight, residence time on the spawning ground, and it is allowed to vary across mothers. In this study, this variation is referred to as parental variation. It should be noted that the magnitude of this parameter $\left(\lambda_{i}\right)$ includes information about the survival rate of the offspring, the number of days after egg hatching, and the egg number; this implies that the parameter reflects the sample timing. It should also be noted that the modeling framework does not depend on whether the reproductive potential is heritable or not.

\section{Nonparental variation}

In addition to parental variation, the variation in reproduction among mothers with the same reproductive potential, referred to here as nonparental variation, is also incorporated into the model, resulting in a large variation in the fertility of the mothers. As the magnitude of the variance increases, the number of successful mothers producing offspring that avoid early life mortality decreases, leading to a situation in which offspring derived from the same mother has highly correlated early life survival probabilities. This situation requires careful consideration of the probability that two offspring share an MHS relationship. Figure 1 presents a schematic representation of the effects of such family-correlated survival on kinship relationships in a population, which are exemplified in iteroparous teleost species. Older mothers are more likely to produce a larger number of offspring, as annual fecundity (i.e., number of eggs, represented by a gray circle) increases with age. However, due to family-correlated survivorship after eggs hatching, the probability that two offspring (i.e., at the larva or juvenile stage, represented by a closed circle) have an MHS relationship is higher (e.g., 53 MHS pairs in Fig. 1b) than in a situation with independent survival (e.g., 32 MHS pairs in Fig. 1a). In other words, MHS pairs have significantly higher or lower collective chances for survival. In addition to family-correlated survivorship, the effects of mating behavior are also incorporated into nonparental variation, such as competition for males/females and correlation between mating opportunities of mother and her offspring number. Nonparental variation may occasionally overshadow the effect of parental variation; however, the average number of offspring per mother is higher for an older mother because the probability of being a successful mother driven by nonparental variation is not biased among mothers.

\section{Distribution of offspring number}

In attempting to incorporate both parental and nonparental variation, it is useful to employ a highly skewed distribution of offspring number. In this study, we use a negative binomial distribution, which is applicable to deviation from the Poisson variance (i.e., overdispersed offspring number with a variance greater than the mean).

Let $k_{i}$ be the number of surviving offspring of mother $i$ at sampling. Given the expected number of offspring $\lambda_{i}, k_{i}$ is 
assumed to follow a negative binomial distribution by a conventional parametrization,

$$
\operatorname{Pr}\left[k_{i} \mid \lambda_{i}\right]=\frac{\Gamma\left[k_{i}+\phi\right]}{k_{i} ! \Gamma[\phi]}\left(\frac{\lambda_{i}}{\phi+\lambda_{i}}\right)^{k_{i}}\left(\frac{\phi}{\phi+\lambda_{i}}\right)^{\phi}
$$

where $\phi(>0)$ is the overdispersion parameter describing the degree of nonparental variation (Akita 2018). At present, $\phi$ is assumed to be constant across mothers, whereas the expected number of surviving offspring $\left(\lambda_{i}\right)$ is variable across mothers. The mean and variance of this distribution are $\lambda_{i}$ and $\lambda_{i}+\lambda_{i}^{2} / \phi$, respectively. In the limit of infinite $\phi$, this distribution becomes a Poisson distribution as follows:

$\lim _{\phi \rightarrow \infty} \operatorname{Pr}\left[k_{i} \mid \lambda_{i}\right]=\frac{\lambda_{i}^{k_{i}} \mathrm{e}^{-\lambda_{i}}}{k_{i} !}$

We assume that $\lambda_{i}$ is independent and identically distributed with a density function $f(\lambda)$, which produces parental variation. The shape of the density function is often complex but may be described by information such as the mother's weight composition in the population. The specific form of $f(\lambda)$ is provided in Appendix A and is used for verifying the theory developed in this paper. As explained in the next subsection, the theory does not require this specific form; it only requires the ratio of the second moment to the squared first moment (i.e., $\mathbb{E}\left[\lambda^{2}\right] / \mathbb{E}[\lambda]^{2}$ ).

\section{MHS probability among randomly selected individuals}

We have derived the approximate probability that two offspring share an MHS relationship with an arbitrary mother (denoted by $\pi$ ) as follows:

$\pi \approx \frac{c}{N+c-1}$

where

$c=\left(1+\phi^{-1}\right) \frac{\mathbb{E}\left[\lambda^{2}\right]}{\mathbb{E}[\lambda]^{2}}$.

The details of the derivation is provided in Appendix B. Equation (3) explicitly contains the two variations (i.e., parental variation and nonparental variation) that determine the degree of deviation from the Poisson distribution. When $\lambda$ is constant across mothers, $\mathbb{E}\left[\lambda^{2}\right]$ equals $\mathbb{E}[\lambda]^{2}$ and then $\pi$ becomes $\left(1+\phi^{-1}\right) /\left(N+\phi^{-1}\right)$, which appears in Eq. (7) in Akita (2018). In addition, as $\phi \rightarrow \infty,\left(1+\phi^{-1}\right) /\left(N+\phi^{-1}\right)$ converges to $1 / N$, which corresponds to the Poisson variance of $k_{i}$ for all mothers in a population. The effect of the two factors causing a deviation from the Poisson distribution can be combined as parameter $c(\geq 1)$. Hereafter, "overdispersion" is referred to as the distribution of the number of offspring resulting from this combined effect.

When $N$ is provided, $\pi$ increases with an increase in $c$, suggesting that a randomly selected pair is more likely to share an MHS relationship under greater overdispersion. Figure S1a-d (Supplementary Information) illustrates the theoretical curve and the simulation results of $\pi$ with $N=$ 100 and 10,000 as a function of $\phi$ or $\mathbb{E}\left[\lambda^{2}\right] / \mathbb{E}[\lambda]^{2}$. This figure demonstrates that the approximation in Eq. (3) works well for the investigated function $f(\lambda)$.

\section{Skewed offspring distribution by parental and nonparental variation}

For illustrative purposes, we demonstrate how parental and nonparental variation skew the offspring distribution in an age-structured population. First, we explore the case in which parental variation is moderately observed and the case in which it is scarcely observed. The cases can be controlled by changing the parameters affecting the shape of $f(\lambda)$. Suppose that the mean fecundity of a mother depends on her age, which can be considered as the reproductive potential. Let $\lambda_{a}$ be mean fecundity, which is a function of age (denoted by $a$ ). Assuming that individual fecundity is proportional to weight and using the von Bertalanffy growth equation for body weight, $\lambda_{a}$ is explicitly described as a function of age as follows:

$\lambda_{a} \propto\left(1-\exp \left[-\kappa\left(a-a_{0}\right)\right]\right)^{\beta}$,

where $\kappa, a_{0}$, and $\beta$ are conventionally used parameters in the von Bertalanffy equation and represent the growth rate, the adjuster of the equation for the initial size of the animal, and the allometric growth parameter, respectively. This relationship indicates that the age distribution generates the variation of $\lambda_{a}$. Given the age distribution, the variation of $f(\lambda)$ increases with $\beta$; meanwhile, when $\beta$ goes to zero, the variation of $f(\lambda)$ vanishes. Figure 2 a presents a histogram of $f(\lambda)$ for the two cases.

Next, we explore $f(\lambda)$ with several combinations of the magnitude of parental and nonparental variations. Figure $2 \mathrm{~b}$, c illustrates the offspring distribution with a relatively low $\beta$ and a moderate $\beta$, respectively. If both parental and nonparental variations are very small, $k$ has as a Poisson distribution (dotted line in Fig. 2b), as noted above. When there is no parental variation, nonparental variation skews the distribution of $k$ (thin and bold lines in Fig. 2b), and vice versa (dotted line in Fig. 2c). In this study, we selected parameter $c=\left(1+\phi^{-1}\right) \mathbb{E}\left[\lambda^{2}\right] / \mathbb{E}[\lambda]^{2}$ to be 1 and 10 for comparison with the results. These two values represent two extreme cases and can be derived from the parameter set $(\phi, \beta)=(1000,0.0009)$ (dotted line in Fig. 2b) and (0.1302, 0.9) (bold line in Fig. 2c), respectively. It should be noted 
(a)

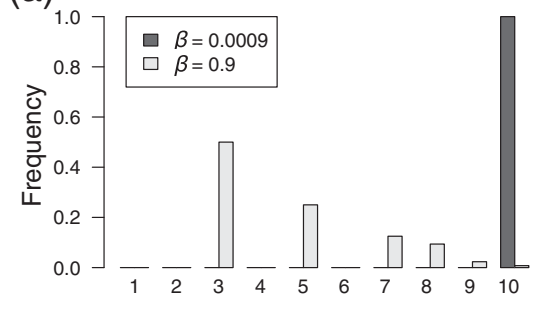

$\lambda$ (b)

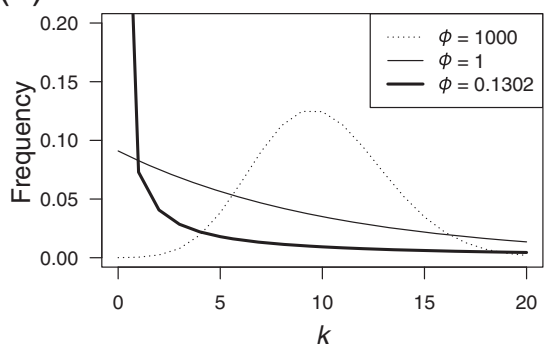

(c)

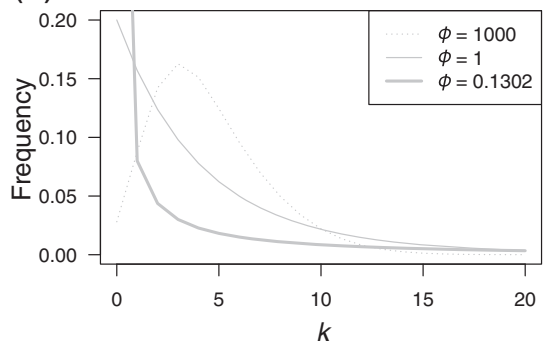

Fig. 2 a Histogram of $f(\lambda)$ assuming fish species with a relatively low $\beta$ (denoted by black bar, $\mathbb{E}\left[\lambda^{2}\right] / \mathbb{E}[\lambda]^{2}=1.0000$ ) and high $\beta$ (denoted by gray bar, $\left.\mathbb{E}\left[\lambda^{2}\right] / \mathbb{E}[\lambda]^{2}=1.1519\right)$. b, c Marginal distribution of $k$ for

that in the latter case, the offspring distribution is highly skewed: $63 \%$ of mothers cannot produce surviving offspring at sampling, and $6 \%$ of mothers produce more than 20 offspring $(\mathbb{E}[\lambda]=4.5$ and $\mathbb{V}[\lambda]=157.5)$. Other parameter values used in $f(\lambda)$ are provided in Appendix A.

\section{Effective mother size and census size}

We have defined the effective mother size as follows:

$$
\begin{aligned}
N_{\mathrm{e}} & =\frac{1}{\pi} \\
& =\frac{N-1}{c}+1 .
\end{aligned}
$$

This definition is similar to the inbreeding effective population size (Nordborg and Krone 2002), as the probability of sharing an MHS relationship $(\pi)$ is identical to the probability that two individuals share a mother in the previous breeding season. It should be noted that when sampling from a single cohort in a population with overlapping generations, the effective mother size in our definition corresponds to the effective breeding mother size, which produces a single cohort.

One might ask whether the proposed effective size is consistent with previous work, such as the drift-based effective population size. When $\lambda$ is constant, the drift-based effective population size is provided as follows:

$$
\begin{aligned}
N_{\mathrm{e}} & =\frac{\lambda^{2}}{\mathbb{V}[k]} N \\
& =\frac{N}{\lambda^{-1}+c-1},
\end{aligned}
$$

where $\mathbb{V}[k]=\lambda+\lambda^{2} / \phi$ and $c=1+\phi^{-1}$. This derivation is based on the natural extension of the existing approach (e.g., Gillespie 2004), relaxing the assumption that mean size of a population does not change (i.e., $\lambda=1$ ). The formulation is similar to the proposed effective size in Eq. (5), but not consistent except for the case of $\lambda=1$. several values of $\phi$ (see legend). $\mathbf{b} \beta=0.0009 ; \operatorname{Pr}[k=0]$ with $\phi=$ 0.1302 equals 0.57 . c $\beta=0.9 ; \operatorname{Pr}[k=0]$ with $\phi=0.1302$ equals 0.63 . Details of $f(\lambda)$ are provided in Appendix A

Using Eq. (5), the ratio of the effective mother size to census size can be written as follows:

$$
\begin{aligned}
\frac{N_{\mathrm{e}}}{N} & =\frac{1}{c}+\frac{1}{N}\left(1-\frac{1}{c}\right) \\
& \approx \frac{1}{c},
\end{aligned}
$$

where $N \gg 1$ is assumed for the purpose of approximation.

\section{Statistical properties of MHS pair number}

In this subsection, given the unconditional probability that two offspring share an MHS relationship (Eq. (3)), we consider the distribution of the number of MHS pairs and its statistical properties. Let $H$ be the number of MHS pairs found in an offspring sample of size $n$. First, we derive the approximate distribution of $H$ for a situation in which overdispersion does not exist (i.e., $c=1$ ). Second, we evaluate whether the derived distribution of $H$ for the nonoverdispersed case is applicable to the overdispersed case (i.e., $c>1$ ).

If overdispersion does not exist (i.e., $c=1$ ), drawing an MHS pair from a randomly selected pair in a sample is considered a Bernoulli trial. Thus, $H$ follows a hypergeometric distribution, which is a function of the sample size of the offspring, the total number of offspring in the population, and the total number of MHS pairs in the population. However, in the setting of this study, the latter two components are random variables, thus creating a complex situation for deriving the exact formulation (Akita 2018). Therefore, assuming that the total number of MHS pairs in the population is much higher than the number of pairs in a sample $\sum_{k} \mathrm{C}_{2} \gg_{n} \mathrm{C}_{2}$ the distribution is approximated by a binomial form as follows:

$$
\operatorname{Pr}\left[H=h \mid n_{\text {pair }}, \pi\right]=\left(\begin{array}{c}
n_{\text {pair }} \\
h
\end{array}\right) \pi^{h}(1-\pi)^{n_{\text {pair }}-h},
$$

where $n_{\text {pair }}$ is the number of pairs in a sample $\left(={ }_{n} \mathrm{C}_{2}\right)$. For practical purposes, the condition $\sum_{k} \mathrm{C}_{2} \gg_{n} \mathrm{C}_{2}$ may be 
acceptable. The theoretical expectation of $H$ is

$\mathbb{E}[H]=n_{\text {pair }} \pi$,

and the variance is

$\mathbb{V}[H]=n_{\text {pair }} \pi(1-\pi)$.

Figure S2a, b (Supplementary Information) illustrates the accuracy of the theoretical prediction for the expectation and the variance of $H$ under the Poisson variance as a function of $n$, respectively. For the investigated parameter, the prediction is demonstrated to be highly accurate.

If overdispersion exists (i.e., $c>1$ ), drawing an MHS pair is no longer a Bernoulli trial. For example, an individual that is born to a relatively successful mother has a greater probability of an MHS relationship with other individuals. Therefore, a hypergeometric/binomial form is not appropriate for the distribution of $H$. As illustrated in Fig. S2d (Supplementary Information), the binomial variance (Eq. (10)) is downwardly biased from the observed variance of $H$ when $n$ increases. The theoretical evaluation is relatively complex and is left for future research. However, for the investigated parameter set, the expected value is well approximated by Eq. (9) (Fig. S2c in Supplementary Information), assuming independent comparisons. The rationale may be that the MHS probability in a pair, $\pi(\mathrm{Eq}$. (3)), includes the effect of overdispersion. Next, on the basis of an accurate approximation of $\mathbb{E}[H]$ in the case of overdispersion, we provide the estimator of $N_{\mathrm{e}}$ from the observed number of MHS pairs in a sample.

\section{Moment estimator of $\mathrm{N}_{\mathrm{e}}$ from observed number of MHS pairs}

By removing $\pi$ in Eqs. (5) and (9), $N_{\mathrm{e}}$ can be written as a function of $c, n_{\text {pair }}$, and $\mathbb{E}[H]$. The observed number of MHS pairs in a sample is defined by $H_{\text {obs }}$, and $\mathbb{E}[H]$ is replaced by $H_{\text {obs }}$, generating the moment estimator of $N_{\mathrm{e}}$ :

$\hat{N}_{\mathrm{e}, 0}=\frac{n_{\text {pair }}}{H_{\text {obs }}}$.

In this paper, a "hat" indicates the estimator of a variable. This relationship can be written as follows:

$$
\left(\frac{\widehat{N-1}}{c}\right)=\frac{n_{\text {pair }}}{H_{\text {obs }}}-1,
$$

indicating that $N$ and $c$ cannot be estimated simultaneously from the number of observed MHS pairs.

Assuming that $H$ follows a binomial distribution, the estimator corresponds to the maximum likelihood estimator of $N_{0}$ (see Appendix C). There are two drawbacks to using this estimator. First, the value of $\hat{N}_{\mathrm{e}, 0}$ becomes inflated when no MHS pairs are observed in a sample (i.e., $H_{\mathrm{obs}}=0$ ). This leads to a situation in which an individual-based model frequently generating zero MHS pairs is not available for statistical evaluation. Second, even if an MHS pair is detected in a sample, it is likely that $\hat{N}_{\mathrm{e}, 0}$ is strongly biased (see Appendix C). Therefore, an improved estimator is necessary for the purpose of appropriate evaluation and higher accuracy for a wide parameter range.

\section{Nearly unbiased estimator of $N_{e}$}

We have derived an alternative estimator of $N_{\mathrm{e}}$ (denoted by $\hat{N}_{\mathrm{e}, 1}$ ) as follows:

$\hat{N}_{\mathrm{e}, 1}=\frac{n_{\text {pair }}+1}{H_{\text {obs }}+1}$.

The derivation process is similar to that of the nearly unbiased estimator of adult number in a population using the mark-recapture method (Chapman 1951), which is based on the idea that the observation of $1 /(H+1)$ approximately provides a linear estimator of $N_{\mathrm{e}}$ (see Appendix D). The bias of $\hat{N}_{\mathrm{e}, 1}$ is defined by $b_{\text {mean }}$, which is given by

$$
\begin{aligned}
b_{\text {mean }} & =\mathbb{E}\left[\hat{N}_{\mathrm{e}, 1}\right]-N_{\mathrm{e}} \\
& =-N_{\mathrm{e}}\left(1-N_{\mathrm{e}}^{-1}\right)^{n_{\text {pair }}+1}
\end{aligned}
$$

It should be noted that $\hat{N}_{\mathrm{e}, 1}$ is downwardly biased; however, this bias may be ignored for a wider range of parameters than $\hat{N}_{\mathrm{e}, 0}$ (see details in the "Results" section), which allows $\hat{N}_{\mathrm{e}, 1}$ to be called a nearly unbiased estimator.

We also determined the estimator of $\mathbb{V}\left[\hat{N}_{\mathrm{e}, 1}\right]$, given by

$\hat{v}=\frac{\left(n_{\text {pair }}+1\right)\left(n_{\text {pair }}-H_{\mathrm{obs}}\right)}{\left(H_{\mathrm{obs}}+1\right)^{2}\left(H_{\mathrm{obs}}+2\right)}$.

The derivation process is similar to that in Seber (1970) (see Appendix $\mathrm{E}$ for details). The bias of $\hat{v}$ is defined by $b_{\mathrm{var}}$, which is given by

$$
\begin{aligned}
b_{\mathrm{var}} & =\mathbb{E}[v]-\mathbb{V}\left[\hat{N}_{\mathrm{e}, 1}\right] \\
& =N_{\mathrm{e}}^{2}\left(\left(1-N_{\mathrm{e}}^{-1}\right)^{n_{\text {pair }}+2}\right. \\
& +\left(\left(n_{\text {pair }}+2\right) N_{\mathrm{e}}^{-1}-2\right)\left(1-N_{\mathrm{e}}^{-1}\right)^{n_{\text {pair }}+1} \\
& \left.+\left(1-N_{\mathrm{e}}^{-1}\right)^{2\left(n_{\text {pair }}+1\right)}\right) .
\end{aligned}
$$

Finally, we consider the estimator of the coefficient of variation of $\hat{N}_{\mathrm{e}, 1}$. A method similar to the derivation of $\hat{v}$ (i.e., searching for a formula such that its expectation approximates $\mathbb{C V}\left[\hat{N}_{\mathrm{e}, 1}\right]$ ) was overly complex for the 
(a)

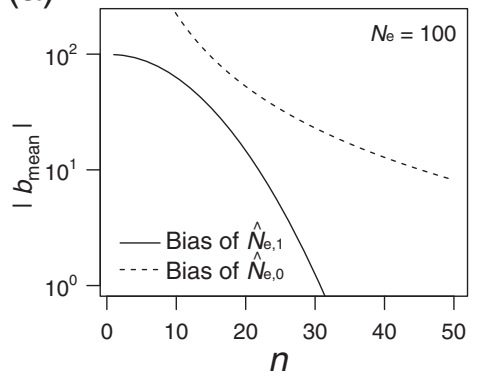

(b)

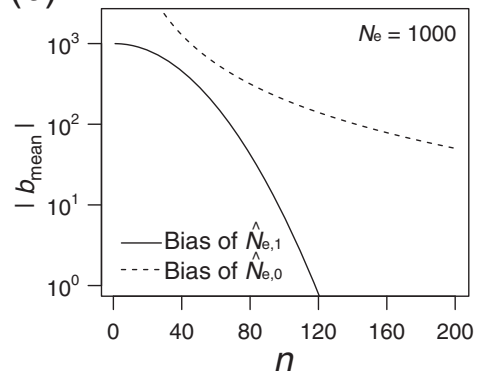

(c)

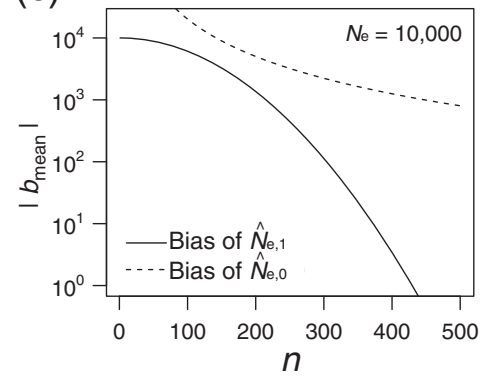

Fig. 3 Absolute bias of $\hat{N}_{\mathrm{e}, 1}$ (represented by a solid line) and $\hat{N}_{\mathrm{e}, 0}$ (represented by a dotted line) as a function of $n$. a $N_{\mathrm{e}}=100 . \mathbf{b} N_{\mathrm{e}}=1000$. c $N_{\mathrm{e}}=$ 10,000

estimator; instead, using Eqs. (13) and (15), we defined the estimator as follows:

$$
\begin{aligned}
\widehat{c v} & =\frac{\sqrt{\hat{v}}}{\hat{N}_{\mathrm{e}, 1}} \\
& =\sqrt{\frac{n_{\mathrm{pair}}-H_{\mathrm{obs}}}{\left(n_{\mathrm{pair}}+1\right)\left(H_{\mathrm{obs}}+2\right)}},
\end{aligned}
$$

Roughly speaking, $\widehat{c v}$ is approximated by $\sqrt{1 /\left(H_{\mathrm{obs}}+2\right)}$ because $n_{\text {pair }} \gg H_{\text {obs }}$, which is similar to an approximate lower bound on the coefficient of variation of $\hat{N}$, as presented in Bravington et al. (2016b).

\section{Individual-based model}

To evaluate the performance of the estimators $\left(\hat{N}_{\mathrm{e}, 1}, \hat{v}\right.$, and $\widehat{c v}$ ), we developed an individual-based model that tracks kinship relationships. The population structure was assumed to be identical to that in the development of the estimators. The population consisted of mothers and their offspring and was assumed to follow a Poisson or negative binomial reproduction. The expected number of surviving offspring of a mother followed the density distribution $f(\lambda)$, which was deterministically specified under stable age structure (see Appendix A). It should be noted that the overdispersion parameter $(c)$ was calculated from $\phi$ and $f(\lambda)$. Each offspring retained the ID of its mother, making it possible to trace an MHS relationship.

Given a parameter set $(N, n, \phi$, and parameters that determine $f(\lambda)$ ), we simulated a population history in which $N$ mothers generated offspring; this process was repeated 100 times. For each history, the sampling process was repeated 1000 times, acquiring 100,000 data points that were used to construct the distribution of $\hat{N}_{\mathrm{e}, 1}$, $\hat{v}$, and $\widehat{c v}$ for each parameter set. $N_{\mathrm{e}}$ was calculated from $N$ and $c($ Eq. (5)).

\section{Temporal method}

To compare the performance between our method and other existing methods, we considered the temporal method, which is based on a moment estimator (Nei and Tajima 1981). The temporal method relies on the temporal changes in allele frequency over time, as information for estimating $N_{\mathrm{e}}$. To calculate the estimator of $N_{\mathrm{e}}$ by the temporal method, simulations were independently run and analyzed.

We evaluated the performance of the temporal method estimator on data simulated under the Wright-Fisher model for a haploid population. For a given $N_{\mathrm{e}}$, the frequency trajectory of 500 independent loci was simulated. For each locus, the maximum number of alleles was set to 10 and initial frequencies of those alleles were fixed to 0.1 at generations 0 . Two samples of $n$ individuals were each randomly taken at generation 0 and 9 from the offspring gene pool (i.e., sampling without replacement). For each combination of parameters $\left(N_{\mathrm{e}}, n\right)$, we run 100,000 replicates and obtained the estimator of $N_{\mathrm{e}}$ (denoted by $\hat{N}_{\mathrm{e}, \mathrm{TM}}$ ) for each replicate. For comparisons, we set an equal sample size at one time for both methods, although the total sampling size of the temporal method was twice that of our method. In the current comparison, we did not consider the case of overdispersion (e.g., $c>1$ ) because an estimation of an extra parameter is needed (see Kitada et al. 2000) and the comparison of the case goes beyond the scope of this work.

\section{Results}

We evaluated the performance of the estimators $\left(\hat{N}_{\mathrm{e}, 1}, \hat{v}\right.$, and $\widehat{c v}$ ) for a situation in which the number of mothers, $N$, and the combined effect of deviation from the Poisson, $c$, were unknown. The parameter values were changed for $N(100$, $1000,10,000$, and 100,000) and $c$ (1 and 10). We primarily addressed the number of samples $(n)$ required to provide 

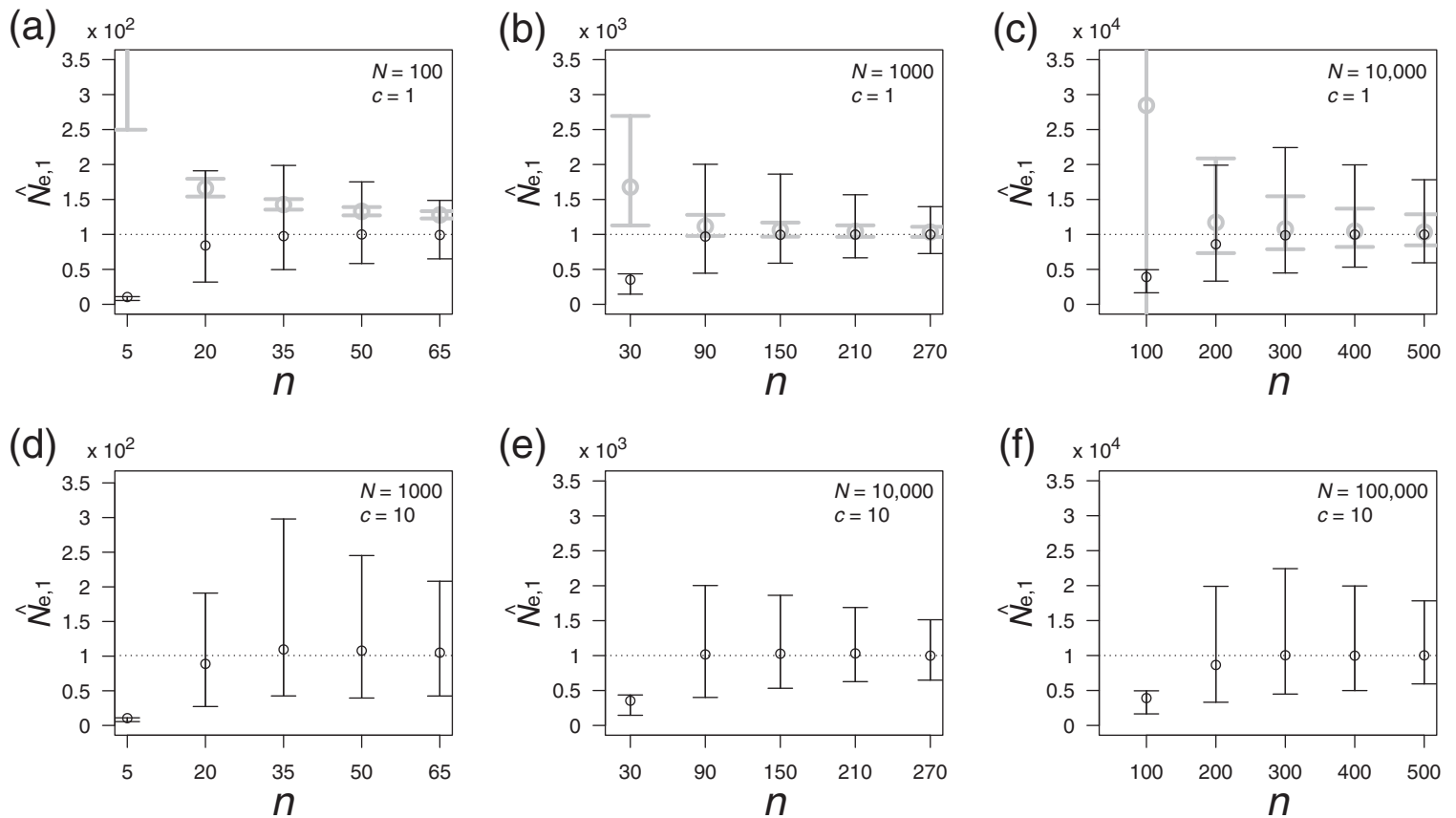

Fig. 4 Accuracy and precision of $\hat{N}_{\mathrm{e}, 1}$ (denoted by black color) and $\hat{N}_{\text {e,TM }}$ (denoted by gray color, only appeared in a-c) as a function of $n$. Open circles represent means with $95 \%$ CIs. A dotted line indicates the true value of $N_{\mathrm{e}}$ which is calculated with given parameters ( $N$ and $c$ ).
$N_{\mathrm{e}} \approx 100$ in $\mathbf{a}$ and $\mathbf{d}, N_{\mathrm{e}} \approx 1000$ in $\mathbf{b}$ and $\mathbf{e}$, and $N_{\mathrm{e}} \approx 10,000$ in $\mathbf{c}$ and $\mathbf{f}$, which are the same value used for calculating $\hat{N}_{\mathrm{e}, \mathrm{TM}}$. The mean value of $\hat{N}_{\mathrm{e}, \mathrm{TM}}$ with $n=5$ in a equals 364 . For illustrative purposes, only a part of the CI in $\hat{N}_{\mathrm{e}, \mathrm{TM}}$ is represented in $\mathbf{a}(n=5)$ and $\mathbf{c}(n=100)$ adequate accuracy and precision under a given parameter set $(N$ and $c)$.

\section{Comparison of the bias of $\hat{N}_{\mathrm{e}, 1}$ with that of $\hat{N}_{\mathrm{e}, 0}$}

First, we evaluated the accuracy of $\hat{N}_{\mathrm{e}, 1}$ based on its bias $\left(b_{\text {mean }}\right)$. For a given $N_{\mathrm{e}}$, the absolute value of the bias is represented by a solid line in Fig. 3a $\left(N_{\mathrm{e}}=100\right), 3 \mathrm{~b}\left(N_{\mathrm{e}}=\right.$ $1000)$, and $3 \mathrm{c}\left(N_{\mathrm{e}}=10,000\right)$ as a function of $n$. For comparison, the bias of $\hat{N}_{\mathrm{e}, 0}$ (see Appendix C) is represented by a dotted line. It is evident that the absolute value of the bias is smaller in $\hat{N}_{\mathrm{e}, 1}$ than in $\hat{N}_{\mathrm{e}, 0}$, because the bias of $\hat{N}_{\mathrm{e}, 1}$ approximately increases with $N_{\mathrm{e}}$ (Eq. (14)) while the bias of $\hat{N}_{\mathrm{e}, 0}$ approximately increases with $\hat{N}_{\mathrm{e}}^{2}$ (Eq. (S13)). There are remarkable differences between them especially in the situation of small sample size $(n)$. Hereafter, we use $\hat{N}_{\mathrm{e}, 1}$ as the estimator of effective mother size.

\section{Accuracy and precision of $\hat{N}_{\mathrm{e}, 1}$}

As expected, $\left|b_{\text {mean }}\right|$ decreases with $n$, as a larger $n$ leads the term $\left(1-N_{\mathrm{e}}^{-1}\right)^{n_{\text {pair }}+1}$ in $b_{\text {mean }}$ to vanish more quickly. The requisite sample size $(n)$ with a small bias of less than $10 \%$ is 22 for $N_{\mathrm{e}}=100\left(\left|b_{\text {mean }}\right|<10\right.$; see Fig. 3a), 69 for $N_{\mathrm{e}}=$ $1000\left(\left|b_{\text {mean }}\right|<100\right.$; see Fig. 3b), and 216 for $N_{\mathrm{e}}=10,000$ ( $b_{\text {mean }} \mid<1000$; see Fig. $3 \mathrm{c}$ ). The results of the individual- based model support the above prediction. Figure 4 illustrates the average value of $\hat{N}_{\mathrm{e}, 1}$ (represented by black open circles) and $\hat{N}_{\mathrm{e}, \mathrm{TM}}$ (represented by gray open circles; details are provided in the next subsection) with a $95 \%$ confidence interval (CI), which is obtained from the individual-based model. As expected, the average value of $\hat{N}_{\mathrm{e}, 1}$ downwardly deviates from $N_{\mathrm{e}}$ for a relatively small sample size $(n)$ satisfying $\left|b_{\text {mean }}\right| \gg 1$. As $n$ increases, the average value of $\hat{N}_{\mathrm{e}, 1}$ approaches a true $N_{\mathrm{e}}$ (represented by a black dotted line in Fig. 4).

Next, we evaluated the precision of $\hat{N}_{\mathrm{e}, 1}$. As illustrated in Fig. 4, the precision of $\hat{N}_{\mathrm{e}, 1}$ for a change in $n$ behaves in a complex manner. For the investigated parameter set, we determined that the degree of precision holds under different combinations of $N$ and $c$ if the value of $N_{\mathrm{e}}$ is fixed $\left(N_{\mathrm{e}} \approx\right.$ $N / c$ equals 100 in Fig. 4a, d, 1000 in Fig. 4b, e, and 10,000 in Fig. 4c, f); this suggests that the level of uncertainty is roughly determined by $N_{\mathrm{e}}$. Although the lower limit of the CI monotonically increases with $n$, the upper limit of the CI has a peak at the point at which the average $\hat{N}_{\mathrm{e}, 1}$ is very close to the true $N_{\mathrm{e}}$. Near this point, the range of the CI is large, and $\hat{N}_{\mathrm{e}, 1}$ is asymmetrically distributed with a longer tail on the large side (e.g., $n=300$ in Fig. 4c, f). As $n$ increases beyond this point, the range of the CI decreases, and the shape of the distribution asymptotically becomes symmetric. 


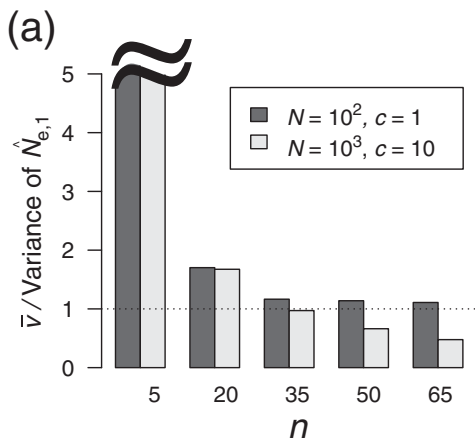

(b)
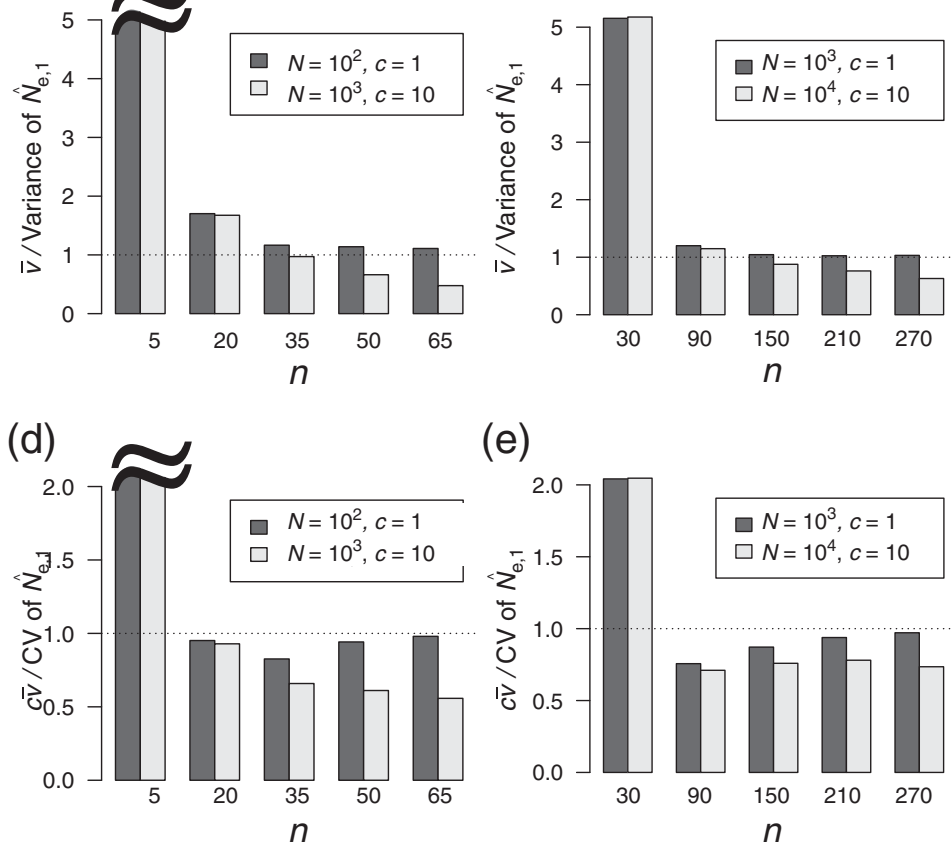

(e)

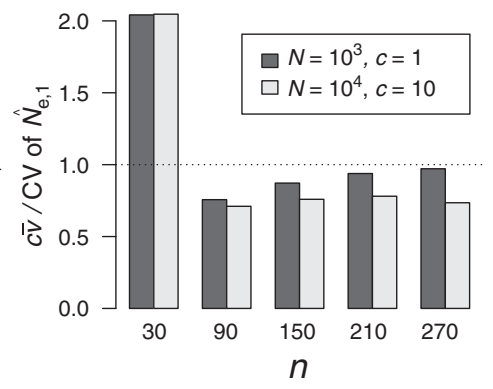

(c)

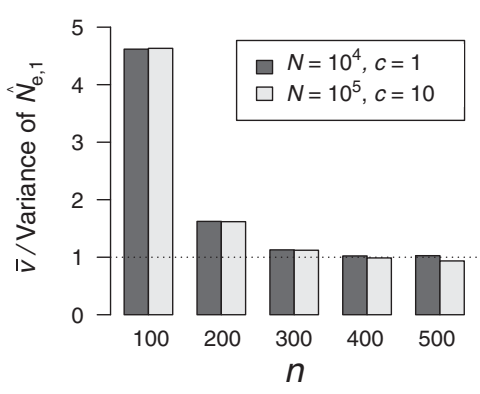

(f)

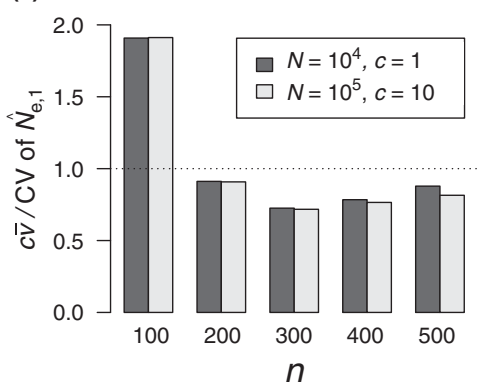

Fig. 5 a-c Ratio of the average $\hat{v}$ to the variance of $\hat{N}_{\mathrm{e}, 1}$ as a function of $n$. d-f Ratio of the average $\widehat{c v}$ to the coefficient of variation of $\hat{N}_{\mathrm{e}, 1}$ as a function of $n$. The value of parameters $\left(N\right.$ and $c$ ) is indicated in the legend. a, $\mathbf{d} N_{\mathrm{e}} \approx 100$. b, e $N_{\mathrm{e}} \approx 1000 . \mathbf{c}, \mathbf{f} N_{\mathrm{e}} \approx 10,000$

\section{Comparison of $\hat{N}_{\mathrm{e}, 1}$ with $\hat{N}_{\mathrm{e}, \mathrm{TM}}$}

As shown in Fig. 4a-c, $\hat{N}_{\mathrm{e}, 1}$ is much more accurate than $\hat{N}_{\text {e,TM }}$ for most of the sample sizes (the number of loci is 500 and the interval generation of sampling is ten; see details in the "Theory" section). In addition, with a relatively small sample size, $\hat{N}_{\mathrm{e}, 1}$ is much more precise than $\hat{N}_{\mathrm{e}, \mathrm{TM}}$. It is generally known that, when $N_{\mathrm{e}}$ is relatively large and sample size is relatively small, the estimated value by the temporal method becomes flawed because the sampling variance overshadows the magnitude of genetic drift that determines the accuracy in the temporal method (Nei and Tajima 1981). Such a situation was confirmed in our setting, as an appearance of negative $\hat{N}_{\mathrm{e}, \mathrm{TM}} \cdot\left(N_{\mathrm{e}}=10,000\right.$ and $n=$ 100; see Fig. 4c). Even when $N_{\mathrm{e}}$ is relatively small, the accuracy of $\hat{N}_{\mathrm{e}, 1}$ is higher than that of $\hat{N}_{\mathrm{e}, \mathrm{TM}}\left(N_{\mathrm{e}}=100\right.$; see Fig. 4a). Together with the theoretical predictions (as shown in Fig. 3), we conclude that the high performance of our method is due to the bias reduction of the estimator, especially in a relatively small sample size.

\section{Accuracy of $\hat{v}$ and $\widehat{c v}$}

We then evaluated the accuracy of $\hat{v}$. Theoretically, the bias of $\hat{v}\left(b_{\text {var }}\right)$ was determined to have a peak at a certain value of $n$, as illustrated in Fig. S3 (Supplementary Information). Figure $5 \mathrm{a}-\mathrm{c}$ presents the ratio of the average $\hat{v}$ to the variance of $\hat{N}_{\mathrm{e}, 1}$ for different combinations of $(N$, c) with fixed $N_{\mathrm{e}}$, which is obtained from the individualbased model. If the ratio is close to $1, \hat{v}$ is deemed an estimator of unbiasedness. When $n$ approaches zero, the ratio becomes inflated (e.g., $n=5$ in Fig. 5a) although $b_{\text {var }}$ also approaches zero (Fig. S3). This inconsistency for a small $n$ (i.e., overestimation) may result from the bias of $\hat{N}_{\mathrm{e}, 1}$. As $n$ increases, the ratio approaches 1 when $c=1$ but less than 1 when $c>1$ ( $c=10$ in Fig. 5a-c), suggesting that the property of unbiasedness holds only under the Poisson variance; however, the degree of this bias is not very high for a relatively large $N_{\mathrm{e}}(c=10$ in Fig. 5c). In other words, the accuracy of $\hat{v}$ is not solely determined by the level of $N_{\mathrm{e}}$. This inconsistency (i.e., underestimation) may result from the assumption that the correlation between pairs can be ignored and thus that the number of HS pairs in the sample follows a binomial distribution (Eq. (8)).

Finally, we evaluated the accuracy of $\widehat{c v}$. Figure $5 \mathrm{~d}-\mathrm{f}$ illustrates the ratio of the average $\widehat{c v}$ to the coefficient of variation of $\hat{N}_{\mathrm{e}, 1}$, which is obtained from the individualbased model. As expected, the property of the estimator is similar to that of $\hat{v}$, as $\widehat{c v}$ is defined by using $\hat{v}$ (Eq. (17)). The ratio becomes inflated for small $n$; as $n$ increases, the ratio approaches 1 when $c=1$ but is $<1$ when $c>1$ (i.e., underestimation); however, the relationship between the degree of bias and the level of $N_{\mathrm{e}}$ is unclear. 


\section{Discussion}

We theoretically developed a nearly unbiased estimator of the number of effective mothers in a population $\left(\hat{N}_{\mathrm{e}, 1}\right)$, the estimator of its variance $(\hat{v})$, and its coefficient of variation $(\widehat{c v})$, which are based on the known MHS relationships found within a single cohort. The performance of the estimators (accuracy and precision) was quantitatively evaluated by running an individual-based model. Our modeling framework allows for two types of reproductive variation; variance of the average offspring number per mother (parental variation) and variance of the offspring number across mothers with the same reproductive potential (nonparental variation). The former is related to age- or size-dependent reproductive potential, whereas the latter is related to family-correlated survival, both of which can result in a skewed distribution of offspring number. These two effects are summarized into one parameter $(c)$ in the framework. Our estimators can be calculated from sample size $(n)$ and the observed number of MHS pairs $\left(H_{\mathrm{obs}}\right)$ and do not require other parameters, such as adult mother size $(N)$ or the degree of overdispersed reproduction $(c)$. The rationale for this is that the observed number of MHS pairs contains information about these parameters.

To estimate the number of effective mothers $\left(N_{\mathrm{e}}\right)$, our theoretical results provide guidance for a sample size to ensure the required accuracy and precision, especially if the order of the number of effective mothers is approximately known. For example, when the effective number of mothers is within $10^{2}-10^{3}$, sampling 50 offspring falls within the range of accuracy of the estimation with a $0-30 \%$ bias (Eq. (14) and Fig. 3). Even if there is no information about the effective number of mothers, the coefficient of variation of the estimated number can be estimated $(\widehat{c v})$ when the sample size is above a given level (Fig. 5c, d). Although the estimator of the variation of the number of mothers $(\hat{v})$ is relatively accurate for the investigated parameter set (Fig. 5a, b), the present estimator of the coefficient of variation is systematically biased; thus, improvements in accuracy are left for future research.

Our modeling framework is presented in the context of the sibship assignment (SA) method, which defined a kinship-oriented estimation of effective population size (Wang 2009; Waples and Waples 2011; Ko and Nielsen 2019). The original theory of the SA method was developed by Wang (2009), and it can perform the estimation of effective population size from HS and FS probabilities, which are calculated by the number of HS and FS pairs in a sample. Wang's estimator reduces to the inverse of the frequency of HS pairs in a sample, which corresponds to $\hat{N}_{\mathrm{e}, 0}$ (Eq. (11)). Our proposed estimator $\hat{N}_{\mathrm{e}, 1}$ (Eq. (13)) is more accurate than $\hat{N}_{\mathrm{e}, 0}$, because the bias of $\hat{N}_{\mathrm{e}, 1}$ approximately increases with $N_{\mathrm{e}}$ (Eq. (14)) while the bias of $\hat{N}_{\mathrm{e}, 0}$ approximately increases with $\hat{N}_{\mathrm{e}}^{2}$ (Eq. (S13)). There are remarkable differences between them specially for small sample sizes, as shown in Fig. 3. In this study, we analytically obtained nearly unbiased estimators $\left(\hat{N}_{\mathrm{e}, 1}, \hat{v}\right.$, and $\left.\widehat{c v}\right)$, although their application is limited to the estimation of effective mother size and the case in which MHS can be perfectly distinguished from PHS and other relatives. The latter limitation may be overcome to some extent by the use of a hypervariable region in the mitochondrial genome and/ or sex-linked markers. It should be noted that genetic differentiation between maternal and paternal relatives is a general problem with pedigree reconstruction (Huisman 2017; Hillary et al. 2018). Therefore, incorporating the uncertainty of differentiation or modifying the theory with the use of HS (not MHS) remains a task for future research.

As a first step in developing unbiased estimators of $N_{\mathrm{e}}$, we examined a relatively simple situation and ignored the complex but important features required in practical scenarios, including errors associated with kinship detection and nonrandom sampling. In general, SAs are biased when only limited molecular information is available (e.g., small number or poor quality of genetic markers), and direction of the biases depends on kinship detection algorithm and how to incorporate prior knowledge into the algorithm. Uncertainties of the proposed estimator of $N_{\mathrm{e}}$ due to limited molecular data could be assessed if an algorithm for SAs is incorporated into our framework. It is expected that if nonrandom sampling is caused by a family-correlated sampling scheme, the effective mother size is underestimated because MHS pairs are more likely to be sampled with this sampling scheme than with random sampling. To reduce this bias, the sampling time and location should be varied, or sampling at an early life stage after hatching should be avoided; this may reduce the effect of family-correlated movement that is not addressed in the current theoretical framework.

Contemporary effective population size can provide not only an understanding of genetic health but also an indication of adult size. If the effect of overdispersion $c$ is invariant across years, $\hat{N}_{\mathrm{e}, 1}$ may behave as an index of the number of mothers per year, making it possible to determine the temporal trends, since Eq. (13) can be rewritten as

$$
\left(\frac{\widehat{N-1}}{c}\right)=\hat{N}_{\mathrm{e}, 1}-1 \text {. }
$$

In this case, the proposed index becomes highly informative, particularly for integrating stock assessment in fisheries management using many types of data (e.g., catch data and abundance index data); this leads to more accurate estimation due to the use of fishery-independent data (Ovenden et al. 2015). Recently, Akita (2018) developed a summary statistic 
that indicates the degree of overdispersion; this statistic is based on the number of MHS pairs and mother-offspring pairs in the sample. The temporal trend of this statistic provides information on whether $c$ is invariant across the years and thus provides criteria for determining whether $\hat{N}_{\mathrm{e}, 1}$ behaves as an index of the number of mothers in a population. It should be noted that, if census size is independently obtained, combined with the estimated effective size, we can estimate the magnitude of reproductive variance and potentially the parameter of nonparental variation (i.e., $\phi$ ), which is generally difficult to obtain and can provide unavailable insights into the underlying ecological processes.

Finally, we note the theoretical connection of our results to the ratio of effective mother size to census size, $N_{\mathrm{e}} / N$. A number of studies have demonstrated that the ratio of the effective size to the census size (including fathers) in highfecundity marine species is estimated to fall within $10^{-3}-10^{-6}$ (Hauser and Carvalho 2008). In our derivation, $N_{\mathrm{e}} / N$ is approximately equal to $1 / c$. If there is only parental variation (i.e., $c=\mathbb{E}\left[\lambda^{2}\right] / \mathbb{E}[\lambda]^{2}=\mathbb{C V}[\lambda]^{2}+1$ ), $c$ cannot have a large value; thus, the ratio cannot become very small (e.g., $<10^{-3}$ ). This theoretical consideration suggests a dominant contribution of nonparental variation to a very small $N_{\mathrm{e}} / N$, which is consistent with the result in Waples (2016).

Acknowledgements The author thanks MV Bravington for motivating me to pursue this research topic. The author thanks A Fujiwara, T Kitakado, M Miyagawa, R Nakamichi, S Nishijima, HS Niwa, H Okamura, O Sakai, M Sekino, A Suda, N Suzuki, and Y Tsukahara for fruitful discussions. The author also thanks $F$ Marandel for sharing a programming code. Finally, the author thanks three anonymous reviewers for constructive feedback that substantially improved the quality of this manuscript. This work was supported by JSPS KAKENHI Grant Number 19K06862.

\section{Compliance with ethical standards}

Conflict of interest The author declares that he has no conflict of interest.

Publisher's note Springer Nature remains neutral with regard to jurisdictional claims in published maps and institutional affiliations.

Open Access This article is licensed under a Creative Commons Attribution 4.0 International License, which permits use, sharing, adaptation, distribution and reproduction in any medium or format, as long as you give appropriate credit to the original author(s) and the source, provide a link to the Creative Commons license, and indicate if changes were made. The images or other third party material in this article are included in the article's Creative Commons license, unless indicated otherwise in a credit line to the material. If material is not included in the article's Creative Commons license and your intended use is not permitted by statutory regulation or exceeds the permitted use, you will need to obtain permission directly from the copyright holder. To view a copy of this license, visit http://creativecommons.org/licenses/by/4.0/.

\section{Appendix}

\section{A. Probability density function and its moment of $\lambda$}

As noted in the main text, our modeling framework does not require the specific form of $f(\lambda)$; instead, it only requires the ratio of the second moment to the squared first moment $\left(\mathbb{E}\left[\lambda^{2}\right] / \mathbb{E}[\lambda]^{2}\right)$. However, the specific form is required for illustrative purposes (Fig. 2a) and for the evaluation of the theoretical results (i.e., calculating the moment or running the individual-based model). Here, we model an age-structured fish population, which serves as a representative example, demonstrating both parental and nonparental variations.

Suppose that the mean fecundity of a mother depends on her age. Let $\lambda_{a}$ be mean fecundity, which is a function of age (denoted by $a$ ). The moment can be described as $\mathbb{E}\left[\lambda^{m}\right]=\sum_{a=0}^{a_{\max }} \lambda_{a}^{m} h_{\text {mat }}(a)$, where $h_{\text {mat }}(a)$ is the frequency of mature mothers at a given age, and $a_{\max }$ is the maximum age. Thus, we can numerically obtain the moment from $\lambda_{a}$ and $h_{\text {mat }}(a)$.

For marine species with a type-III survivorship curve, it is generally assumed that individual fecundity is proportional to weight. Using the von Bertalanffy growth equation for body weight, $\lambda_{a}$ is explicitly described as a function of age as follows (identical to Eq. (4)):

$\lambda_{a} \propto\left(1-\exp \left[-\kappa\left(a-a_{0}\right)\right]\right)^{\beta}$,

where $\kappa, a_{0}$, and $\beta$ are conventionally used parameters in the von Bertalanffy equation and represent the growth rate, the adjuster of the equation for the initial size of the animal, and the allometric growth parameter, respectively. For obtaining a specific value of $\lambda$, a coefficient value of 10 multiplied by the right-hand side of Eq. (4) was used when running the individual-based model.

The frequency of mature mothers at a given age can be written as follows:

$h_{\text {mat }}(a) \propto h(a) Q(a)$,

satisfying $\sum_{a=0}^{a_{\max }} h_{\mathrm{mat}}(a)=1$, where $h(a)$ and $Q(a)$ represent the frequency and maturity at a given age, respectively. Although $f(a)$ is affected by historical population dynamics and age-dependent survival, for simplicity, the mortality rate is assumed to be constant (i.e., age independent):

$h(a) \propto\left\{\begin{array}{ll}S^{a} & \text { if } a<a_{\max } \\ 0 & \text { if } a=a_{\max }\end{array}\right.$, 
where $S$ is survival probability. Maturity at age $(Q(a))$ is assumed to be a knife-edge function, given by

$Q(a)=\left\{\begin{array}{l}1 \text { if } a \geq a_{\mathrm{mat}} \\ 0 \text { otherwise }\end{array}\right.$

where $a_{\text {mat }}$ is the mature age

For calculating $\mathbb{E}\left[\lambda^{2}\right] / \mathbb{E}[\lambda]^{2}$, the required parameter set is $\left(a_{\mathrm{max}}, \kappa, a_{0}, \beta, S, a_{\mathrm{mat}}\right)$. In this paper, for the purpose of representation, we fixed the value of several parameters as follows: $a_{\max }=20, \kappa=0.3, a_{0}=0, S=0.5$ and $a_{\text {mat }}=0$. In addition, we selected parameter value $c\left(=\left(1+\phi^{-1}\right)\right.$ $\left.\mathbb{E}\left[\lambda^{2}\right] / \mathbb{E}[\lambda]^{2}\right)$ to be 1 and 10 for comparison with the results in the main text, which are derived from the parameter set $(\phi, \beta)=(1000,0.0009)$ and $(0.1302,0.9)$, respectively.

Finally, we provide the specific forms of $f(\lambda)$ and $\operatorname{Pr}[k]$, which are presented in the main text (Fig. 2). When $\lambda_{a}$ and $h_{\text {mat }}(a)$ are obtained, $f(\lambda)$ is given by

$f(\lambda)=\left\{\begin{array}{ll}h_{\mathrm{mat}}(a) & \text { if } \lambda=\lambda_{a} \\ 0 & \text { otherwise }\end{array}\right.$.

Using Eqs. (1) and (S 4), we can obtain the specific form of the marginal distribution of $k$ by

$\operatorname{Pr}[k]=\sum_{\lambda} \operatorname{Pr}[k \mid \lambda] f(\lambda)$.

\section{B. Probability that two offspring share an MHS relationship}

Given the realized number of offspring $k_{1}, k_{2}, \ldots, k_{N}$, the probability that two randomly selected offspring are born to mother $i$ is $k_{i} / \sum_{j=1}^{N} k_{j} \times\left(k_{i}-1\right) /\left(\sum_{j=1}^{N} k_{j}-1\right)$. Thus, the conditional probability that two offspring share an MHS relationship with an arbitrary mother is

$$
\begin{aligned}
\pi \mid k_{1}, \ldots, k_{N} & =\sum_{i=1}^{N} \frac{k_{i}\left(k_{i}-1\right)}{\left(\sum_{j=1}^{N} k_{j}\right)\left(\sum_{j=1}^{N} k_{j}-1\right)} \\
& =\frac{\sum_{i=1}^{N} k_{i}^{2}-\sum_{i=1}^{N} k_{i}}{\left(\sum_{j=1}^{N} k_{j}\right)^{2}-\sum_{j=1}^{N} k_{j}} .
\end{aligned}
$$

It should be noted that $k_{i}$ is a random variable followed by a negative binomial distribution (Eq. (1)), in which the parameter of the distribution, $\lambda_{i}$, is also a random variable followed by an arbitral function $f(\lambda)$. By taking the expectation over the distribution of offspring number, the conditional probability is given by

$$
\begin{aligned}
\pi \mid \lambda_{1}, \ldots, \lambda_{N} & =\mathbb{E}\left[\pi \mid k_{1}, \ldots, k_{N}\right] \\
& \approx \frac{\sum_{i=1}^{N} \mathbb{E}\left[k_{i}^{2} \mid \lambda_{i}\right]-\sum_{i=1}^{N} \mathbb{E}\left[k_{i} \mid \lambda_{i}\right]}{\mathbb{E}\left[\left(\sum_{j=1}^{N} k_{j}\right)^{2} \mid \lambda_{1}, \ldots, \lambda_{N}\right]-\sum_{j=1}^{N} \mathbb{E}\left[k_{j} \mid \lambda_{j}\right]} \\
& =\frac{\sum_{i=1}^{N}\left(1+\phi^{-1}\right) \lambda_{i}^{2}}{\sum_{i=1}^{N}\left(1+\phi^{-1}\right) \lambda_{i}^{2}+2 \sum_{i>j} \lambda_{i} \lambda_{j}}
\end{aligned}
$$

From the first to the second line, we use the approximation that $\mathbb{E}\left[g_{1}(k) / g_{2}(k)\right] \approx \mathbb{E}\left[g_{1}(k)\right] / \mathbb{E}\left[g_{2}(k)\right]$. The expectations are averaged over $k$ or $k^{2}$, conditional on $\lambda$. By taking the expectation over $\lambda$ and applying a similar approximation, the unconditional probability is given by

$$
\begin{aligned}
\pi & =\mathbb{E}\left[\pi \mid \lambda_{1}, \ldots, \lambda_{N}\right] \\
& \approx \frac{\left(1+\phi^{-1}\right) \mathbb{E}\left[\lambda^{2}\right]}{\left(1+\phi^{-1}\right) \mathbb{E}\left[\lambda^{2}\right]+(N-1)(\mathbb{E}[\lambda])^{2}} .
\end{aligned}
$$

This provides the formulation described in Eq. (3). In computing the expectation, we remove the subscript ( $i$ or $j$ ) because $\lambda$ is independent and identically distributed.

\section{Properties of moment estimator of $\mathbf{N}$}

Here, we demonstrate that $\hat{N}_{\mathrm{e}, 0}$ in Eq. (11) is the maximum likelihood estimator and that $\hat{N}_{\mathrm{e}, 0}$ is upwardly biased, especially when $n$ is small. Let $L$ be the likelihood of the distribution of $H$ (Eq. (8)). Given the observation (i.e., $H_{\mathrm{obs}}$ ), the partial derivative of the log-likelihood with respect to $\pi$ is given by

$\frac{\partial \ln L}{\partial \pi} \propto \frac{H_{\mathrm{obs}} n_{\mathrm{pair}}}{\pi}-\frac{\left(n_{\mathrm{pair}}-H_{\mathrm{obs}}\right) n_{\mathrm{pair}}}{1-\pi}$,

leading to the maximum likelihood estimator of $\pi$ :

$\hat{\pi}=\frac{H_{\mathrm{obs}}}{n_{\mathrm{pair}}}$,

where $\hat{\pi}$ satisfies $\left.(\partial \ln L / \partial \pi)\right|_{\pi=\hat{\pi}}=0$. Substituting Eq. (3) into Eq. (S10), we can obtain the estimator $\left(\hat{N}_{\mathrm{e}, 0}\right)$ described in Eq. (11).

Consider the bias of $\hat{N}_{0}$ defined by $\mathbb{E}\left[\hat{N}_{\mathrm{e}, 0}\right]-N_{\mathrm{e}}$. We set the following equations:

$$
\begin{aligned}
g(H) & =\hat{N}_{\mathrm{e}, 0} \\
& =\frac{n_{\text {pair }}}{H},
\end{aligned}
$$


and

$$
\begin{aligned}
g(\mu) & =N_{\mathrm{e}} \\
& =\frac{n_{\text {pair }}}{\mu},
\end{aligned}
$$

where $\mu=\mathbb{E}[H]$. Using Eqs. (9) and (10), the bias is given by

$$
\begin{aligned}
\mathbb{E}[g(H)-g(\mu)] & \approx \frac{1}{2} \mathbb{E}\left[g^{\prime \prime}(H)(H-\mu)^{2}\right] \\
& =\frac{1}{2} \frac{2 n_{\text {pair }}}{\mu^{3}} \mathbb{V}[H] \\
& \approx \frac{N_{\mathrm{e}}^{2}}{n_{\text {pair }}}
\end{aligned}
$$

where a quadratic approximation for $g$ centered at $\mu$ and $\mathbb{V}[H] \approx \mathbb{E}[H]$ is used. The value of the right-hand side of Eq. (S13) is illustrated in Fig. $3 \mathrm{a}, \mathrm{b}$ as the bias of $\hat{N}_{\mathrm{e}, 0}$.

\section{Derivation of nearly unbiased estimator of $N_{e}$}

We consider the following

$$
\begin{aligned}
\mathbb{E}\left[\frac{1}{H+1}\right] & =\sum_{h=0}^{n_{\text {pair }}} \frac{1}{h+1} \operatorname{Pr}\left[h \mid n_{\text {pair }}\right] \\
& =\frac{1}{\left(n_{\text {pair }}+1\right) \pi} \sum_{h^{\prime}=1}^{n_{\text {pair }}+1} \operatorname{Pr}\left[h^{\prime} \mid n_{\text {pair }}+1\right] \\
& =\frac{1}{\left(n_{\text {pair }}+1\right) \pi}\left(1-\operatorname{Pr}\left[h^{\prime}=0 \mid n_{\text {pair }}+1\right]\right) \\
& =\frac{1-(1-\pi)^{n_{\text {pair }}}+1}{\left(n_{\text {pair }}+1\right) \pi},
\end{aligned}
$$

assuming the binomial form of $H$ (Eq. (8)). Equation (S14) is not directly applied for the derivation of the estimator of $N_{\mathrm{e}}$ due to the complex formulation. Thus, we simplify the formulation as follows:

$\mathbb{E}\left[\frac{1}{H+1}\right] \approx \frac{1}{\left(n_{\text {pair }}+1\right) \pi}$

assuming that

$$
(1-\pi)^{n_{\text {pair }}+1} \approx 0 .
$$

This simplification deviates from the prediction by Eq. (S14) when $n$ is relatively small. Replacing $\mathbb{E}[1 /(H+1)]$ by $1 /\left(H_{\mathrm{obs}}+1\right)$ in the left-hand side of Eq. (S15), we can obtain the estimator $\left(\hat{N}_{\mathrm{e}, 1}\right)$ described in Eq. (13).

For the evaluation of $\hat{N}_{\mathrm{e}, 1}$, the bias is calculated. $\mathbb{E}\left[\hat{N}_{\mathrm{e}, 1}\right]$ is required for the calculation and given by

$$
\begin{aligned}
\mathbb{E}\left[\hat{N}_{\mathrm{e}, 1}\right] & =\left(n_{\text {pair }}+1\right) \mathbb{E}\left[\frac{1}{H+1}\right] \\
& =N_{\mathrm{e}}-N_{\mathrm{e}}\left(1-N_{\mathrm{e}}^{-1}\right)^{n_{\text {pair }}+1},
\end{aligned}
$$

where the relationship in Eq. (S14) is used. This provides the formulation of the bias, as described in Eq. (14).

\section{E. Derivation of estimator of variance of $\hat{N}_{\mathrm{e}, 1}$}

Let $v$ be the estimator of the variance of $\hat{N}_{\mathrm{e}, 1}$. It is desirable for $v$ to be defined such that the bias (denoted $b_{\text {var }}$ ) is reasonably small. From Eq. (13), the variance of $\hat{N}_{\mathrm{e}, 1}$ is given by

$$
\begin{aligned}
\mathbb{V}\left[\hat{N}_{\mathrm{e}, 1}\right] & =\left(n_{\text {pair }}+1\right)^{2} \mathbb{V}\left[\frac{1}{H+1}\right] \\
& =\left(n_{\text {pair }}+1\right)^{2}\left(\mathbb{E}\left[\frac{1}{(H+1)^{2}}\right]-\mathbb{E}\left[\frac{1}{(H+1)}\right]^{2}\right) \\
& =\mathbb{E}\left[\frac{\left(n_{\text {pair }}+1\right)^{2}}{(H+1)^{2}}\right]-N_{\mathrm{e}}^{2}\left(1-\left(1-N_{\mathrm{e}}^{-1}\right)^{n_{\text {pair }}+1}\right)^{2}
\end{aligned}
$$

where the term $\mathbb{E}[1 /(H+1)]$ is calculated from the relationship in Eq. (S14). Roughly speaking, $\mathbb{V}\left[\hat{N}_{\mathrm{e}, 1}\right]$ is dominated by two terms when $n_{\text {pair }}$ is relatively large: $\mathbb{E}\left[\left(n_{\text {pair }}+1\right)^{2} /(H+1)^{2}\right]$ and $-\hat{N}_{\mathrm{e}}^{2}$. Thus, it is expected that $\mathbb{E}[v]$ includes both terms for a reasonably small bias. We propose the following formulation for $v$ :

$$
\begin{aligned}
v & =\frac{\left(n_{\text {pair }}+1\right)\left(n_{\text {pair }}-H\right)}{(H+1)^{2}(H+2)} \\
& =\frac{\left(n_{\text {pair }}+1\right)^{2}}{(H+1)^{2}}-\frac{\left(n_{\text {pair }}+1\right)\left(n_{\text {pair }}+2\right)}{(H+1)(H+2)} .
\end{aligned}
$$

The expectation of the second term in Eq. (S19) is given by $\mathbb{E}\left[\frac{\left(n_{\text {pair }}+1\right)\left(n_{\text {pair }}+2\right)}{(H+1)(H+2)}\right]$

$=\left(n_{\text {pair }}+1\right)\left(n_{\text {pair }}+2\right) \sum_{h=0}^{n_{\text {pair }}} \frac{1}{(h+1)(h+2)} \operatorname{Pr}\left[h \mid n_{\text {pair }}\right]$ 


$$
\begin{aligned}
& =\frac{1}{\pi^{2}} \sum_{h^{\prime}=2}^{n_{\text {pair }}+2} \operatorname{Pr}\left[h^{\prime} \mid n_{\text {pair }}+2\right] \\
& =N_{\mathrm{e}}^{2}\left(1-\operatorname{Pr}\left[h^{\prime}=0 \mid n_{\text {pair }}+2\right]-\operatorname{Pr}\left[h^{\prime}=1 \mid n_{\text {pair }}+2\right]\right) \\
& =N_{\mathrm{e}}^{2}\left(1-\left(1-N_{\mathrm{e}}^{-1}\right)^{n_{\text {pair }}+2}-\left(n_{\text {pair }}+2\right) N_{\mathrm{e}}^{-1}\left(1-N_{\mathrm{e}}^{-1}\right)^{n_{\text {pair }}+1}\right),
\end{aligned}
$$

leading to a relatively small $b_{\mathrm{var}}$ when $n_{\text {pair }}$ is large, which is described in Eq. (16).

\section{References}

Akita T (2018) Statistical test for detecting overdispersion in offspring number based on kinship information. Popul Ecol 60:297-308

Bravington MV, Grewe PM, Davies CR (2016a) Absolute abundance of southern bluefin tuna estimated by close-kin mark-recapture. Nat Commun 7:13162

Bravington MV, Skaug HJ, Anderson EC et al. (2016b) Close-kin mark-recapture. Stat Sci 31:259-274

Chapman DG (1951) Some properties of hypergeometric distribution with application to zoological census. Univ Calif Public Stat $1: 131-160$

Eldon B, Riquet F, Yearsley J, Jollivet D, Broquet T (2016) Current hypotheses to explain genetic chaos under the sea. Curr Zool 62:551-566

Felsenstein J (1971) Inbreeding and variance effective numbers in populations with overlapping generations. Genetics 68:581-597

Gillespie JH (2004) Population genetics: a concise guide. John Hopkins University Press, Baltimore

Hauser L, Carvalho GR (2008) Paradigm shifts in marine fisheries genetics: ugly hypotheses slain by beautiful facts. Fish Fish 9:333-362

Hedgecock D, Pudovkin AI (2011) Sweepstakes reproductive success in highly fecund marine fish and shellfish: a review and commentary. B Mar Sci 87:971-1002

Hillary RM, Bravington MV, Patterson TA, Grewe P, Bradford R, Feutry P et al. (2018) Genetic relatedness reveals total population size of white sharks in eastern australia and new zealand. Sci Rep $8: 2661$

Huisman J (2017) Pedigree reconstruction from SNP data: parentage assignment, sibship clustering and beyond. Mol Ecol Resour 17:1009-1024
Kitada S, Hayashi T, Kishino H (2000) Empirical bayes procedure for estimating genetic distance between populations and effective population size. Genetics 156:2063-2079

Ko A, Nielsen R (2019) Joint estimation of pedigrees and effective population size using markov chain monte carlo. Genetics 212:855-868

Luikart G, Ryman N, Tallmon DA, Schwartz MK, Allendorf FW (2010) Estimation of census and effective population sizes: the increasing usefulness of DNA-based approaches. Conserv Genet 11:355-373

Marandel F, Lorance P, Berthelé O, Trenkel VM, Waples RS, Lamy JB (2018) Estimating effective population size of large marine populations, is it feasible? Fish Fish 20:189-198

Nei M, Tajima F (1981) Genetic drift and estimation of effective population size. Genetics 98:625-640

Nomura T (2008) Estimation of effective number of breeders from molecular coancestry of single cohort sample. Evol Appl $1: 462-474$

Nordborg M, Krone SM (2002) Separation of time scales and convergence to the coalescent in structured populations. Modern developments in theoretical population genetics: the legacy of Gustave Malécot. Oxford University Press, Oxford, p 194-232

Ovenden JR, Berry O, Welch DJ, Buckworth RC, Dichmont CM (2015) Ocean's eleven: a critical evaluation of the role of population, evolutionary and molecular genetics in the management of wild fisheries. Fish Fish 16:125-159

Pudovkin AI, Zaykin DV, Hedgecock D (1996) On the potential for estimating the effective number of breeders from heterozygoteexcess in progeny. Genetics 144:383-387

Seber GAF (1970) The effects of trap response on tag recapture estimates. Biometrics 26:13-22

Skaug HJ (2017) The parent-offspring probability when sampling agestructured populations. Theor Popul Biol 118:20-26

Tellier A, Lemaire C (2014) Coalescence 2.0: a multiple branching of recent theoretical developments and their applications. Mol Ecol 23:2637-2652

Wang J (2009) A new method for estimating effective population sizes from a single sample of multilocus genotypes. Mol Ecol 18:2148-2164

Wang J, Santiago E, Caballero A (2016) Prediction and estimation of effective population size. Heredity 117:193-206

Waples RS (2006) A bias correction for estimates of effective population size based on linkage disequilibrium at unlinked gene loci. Conserv Genet 7:167

Waples RS (2016) Tiny estimates of the $N_{\mathrm{e}} / N$ ratio in marine fishes: are they real? J Fish Biol 89:2479-2504

Waples RS, Waples RK (2011) Inbreeding effective population size and parentage analysis without parents. Mol Ecol Resour $11: 162-171$ 